\title{
Radiofrequency method for measurement of liquefied petroleum gas mass in the tank of a gas tank truck
}

\author{
Alexander Sovlukov ${ }^{1, *}$ and Victor Tereshin ${ }^{2}$ \\ ${ }^{1}$ V.A. Trapeznikov Institute of Control Sciences of Russian Academy of Sciences, 65, \\ Profsoyuznaya str., 117997, Russia \\ ${ }^{2}$ Technosensor Ltd, 2, Blagodatnaya str., Saint Petersburg, 196128, Russia
}

\begin{abstract}
Radiofrequency method for measurement of liquefied petroleum gas mass under overload from gas tank trucks into tanks of automotive gas-filling stations and gas-holders is considered. Experience of application of radiofrequency measurement system SU-5D-MASS for mass measurement of various liquids and gas condensates under their storage in reservoirs and pumping through pipelines is taken into account. Optimization of measurement system is suggested implying equipment of 1-2 gas tank-cars by Coriolis flow-meters and radiofrequency sensor of liquefied petroleum gas mass in the tank of a gas tank-car while other gas tank-cars are equipped only such a mass sensor. New approach of calibration of tanks of gas tank trucks and automotive gas-filling stations is suggested.
\end{abstract}

\section{Introduction}

Liquefied petroleum gas (LPG) is the mixture of propane and butane where a small quantities of methane, ethane and other components are presented [1,2]. Density of such a substance depends both on temperature and LPG component composition. Compositions of LPG gaseous and liquid phases are differen. LPG composition and density are changed under the change of temperature and overload.

LPG is produced at oil-processed plants and gas-condensate fields. LPG is transported in railway tanks with volumes of $54 \mathrm{~m}^{3}$ and $85 \mathrm{~m}^{3}$, in tanks of gas tank-cars with volumes up to $65 \mathrm{~m}^{3}$ and in sea 20 -foot tanks with volumes of $25 \mathrm{~m}^{3}$. LPG is transported on big distances from points of its production to storage bases - gas-filling stations and storehouses of LPG producers by railway tanks. LPG is supplied from storage bases to gas tank trucks. In tanks of gas tank trucks and sea tanks LPG is transported directly to consumers (automotive gas-filling stations, group reservoir installations, gas-holders of autonomous gas-supply, boilers) and is transferred them according to their requests. A gas tank truck delivers LPG to several consumers as a rule.

Accurate calculation of LPG in transport tanks is very important at the whole way of LPG transport from a producer to an end consumer. Accurate data on LPG mass in a gas

*Corresponding author: sovlukov@yandex.ru 
tank truck and also received into it and overloaded from it gives ability to have full counting of LPG and to exclude ability of non-sanctioned LPG pouring off from a gas tank.

For measurement of LPG mass in reservoirs indirect method of static measurements is often used. In this case LPG level and density are measured, then volume and mass are calculated by multiplication of volume and density using calibration table [1-3]. Measuring systems based on this method don't provide a needed accuracy because of channels for density measurement and not reliable operation of float level and density meters at low values of a liquid density. LPG density may change from $460 \mathrm{~kg} / \mathrm{m}^{3}$ to $630 \mathrm{~kg} / \mathrm{m}^{3}$.

Quantity of light oil products in transport tanks may be determined by a ruler but this way is not suitable for LPG because it is present in a tank under pressure up to 1,6 MPa.

Radiofrequency (RF) measurement method and RF measuring devices were designed for highly accurate measurement of LPG technological parameters [4,5]. Direct method of static LPG mass measurement via application of RF sensors is realized in the measuring system SU-5D with RF sensors DZhS-7M [4-7]. Output parameter of the sensor is LPG total mass including mass of LPG gaseous phase.

\section{Radiofrequency measuring system SU-5D-MASS}

The measuring system SU-5D-MASS (Fig. 1) is used for commercial counting of LPG in gas tank trucks and in gas filling stations. Namely measurement and calculation of the following parateters: total LPG mass in a reservoir; mass of LPG liquid phase; mass of LPG vapour (gaseous) phase; densities of LPG liquid and gaseous phases; LPG level; LPG volume; LPG temperature (6 measurement points); pressure. Error of LPG total mass measurement (mass of LPG liquid and gaseous phases) is not more than $\pm 0,65 \%$.

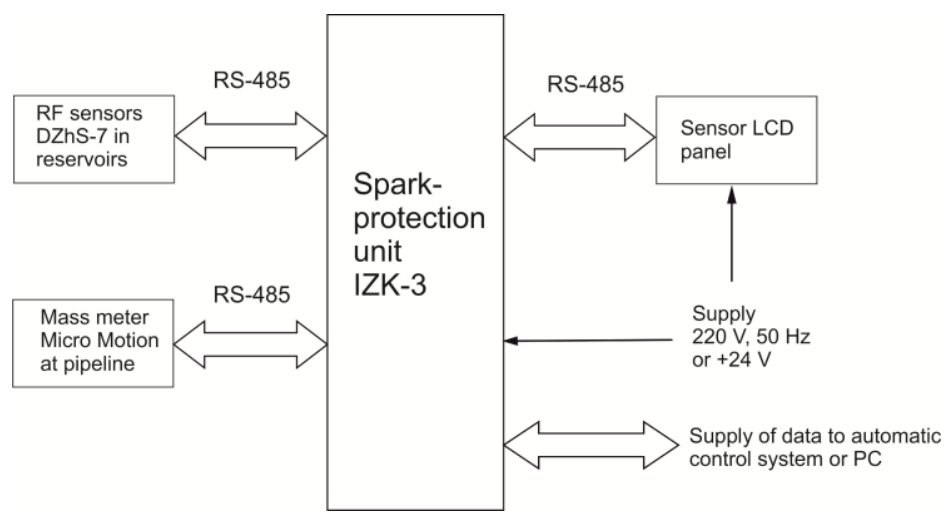

Fig. 1. Structural scheme of the measuring system.

In minimal configuration LPG mass measurement in a tank of gas tank truck is provided via application of RF sensor DZhS-7m or DZhS-7MB depending on the type of a reservoir (Fig. 2). Such sensor is connected to the spark-plug unit IZK-3 containing system unit SB-5 (Fig. 3). Measurement of overloaded LPG mass is done using Coriolis mass-meters on pipelines of liquid and gaseous phases of LPG. Data from all the objects comes on-line to a server, archives and reports are done. It gives ability to have full LPG counting: LPG mass overloaded from gas-filling station must be equal to the mass come into a gas tank truck, LPG mass received at an automotive gas-station must be equal to the LPG mass poured from the gas tank truck.

All the calculations are fulfilled in the spark-plug unit IZK-3 that is positioned in the system unit SB-5. Data from it come to PC, sensor panel and other devices by standard 
protocol Modbus. It is automatically determined what temperature sensors are positioned in LPG liquid phase and in vapour (above the liquid) phase; averaged temperture values of liquid and vapour are calculated.

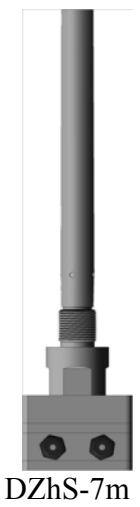

Fig. 2. Sensors.

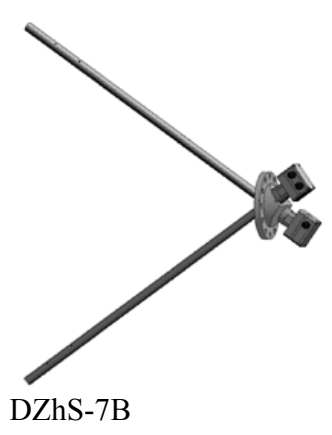

DZhS-7B

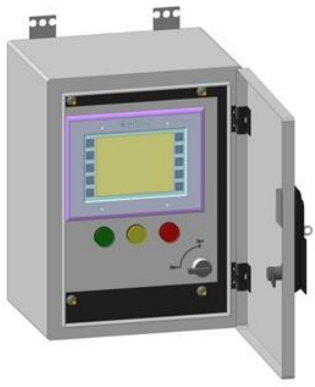

Fig. 3. System unit SB-5.

\section{Radiofrequency method of LPG mass measurement}

In a gas tank truck a reservoir has circular form and is horizontally positioned (Fig. 4). Locations of the sensors - of coaxial RF-sensor and of temperature sensors (6 sensors) in a reservoir are shown in Fig. 4. Here $\mathrm{H}$ - LPG level; $\mathrm{L}_{1}$ - length of RF-sensor; L - distance from the edge of a hatch to the bottom of a tank; $\mathrm{X}_{0}=\mathrm{L}-\mathrm{L}_{1}-$ distance from the bottom to the lower end of the sensor; $\mathrm{L}_{0}-$ LPG level under filling in $100 \%$ of the volume of a tank; $h-$ level of filling in of the sensor by a monitored substsance; $t_{1}, t_{2}, t_{3}, t_{4}, t_{5}, t_{6}$ - location points of temperature sensors.

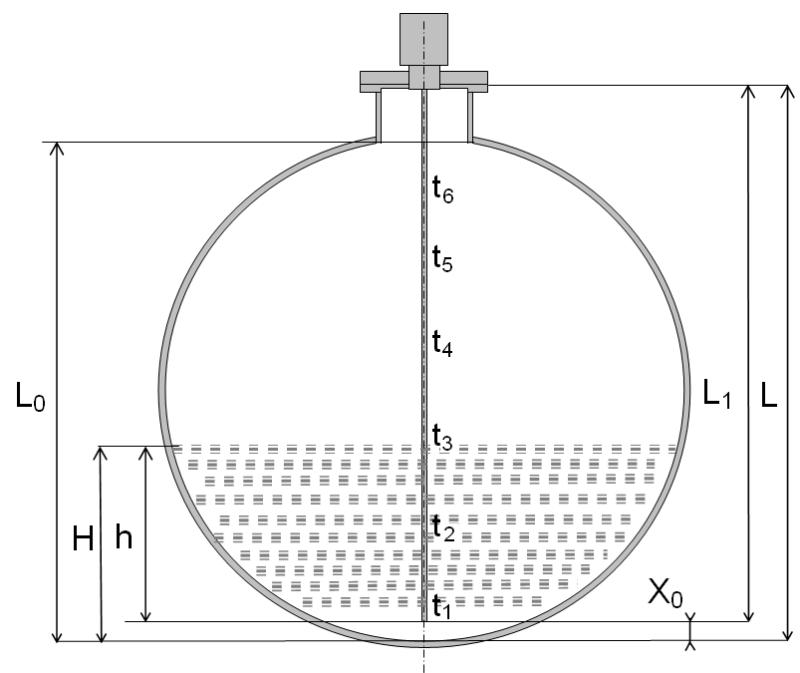

Fig. 4. Position of sensors in a reservoir.

Liquefied petroleum gases are dielectrics, their dielectric permittivity depends on LPG composition and temperature. Dielectric permittivity of LPG is proportional to its density [8]. Therefore electrical capacitance of RF sensor is proportional to the result of multiplication of level and density. RF sensor is really the device for LPG mass measurement. 


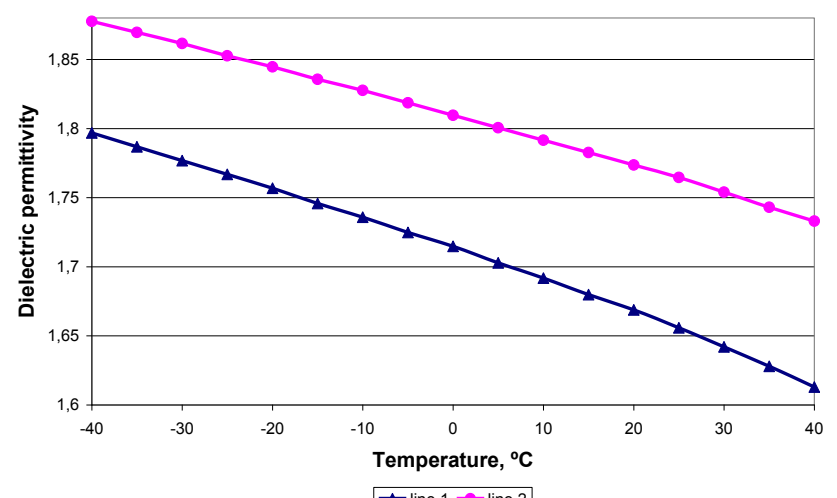

Fig. 5. Temperature dependence of dielectric permittivity for propane (line 1) and butane (line 2).

Diagrams for temperature dependencies of dielectric permittivity for propane (line 1) and butane (line 2) are shown in Fig. 5. Modeling results for temperature error of LPG mass measurement in measuring system with RF sensor tuned for propane mass measurement are in Fig. 6: line 1 - error of propane mass measurement without temperature correction; line 2 - error of butane mass measurement without temperature correction; line 3 - error of propane mass measurement with temperature correction $+0,06 \%$ per $1{ }^{\circ} \mathrm{C}$; line 4 - error of butane mass measurement with temperature correction $+0,06 \%$ per $1{ }^{\circ} \mathrm{C}$ (correction by temperature for propane).

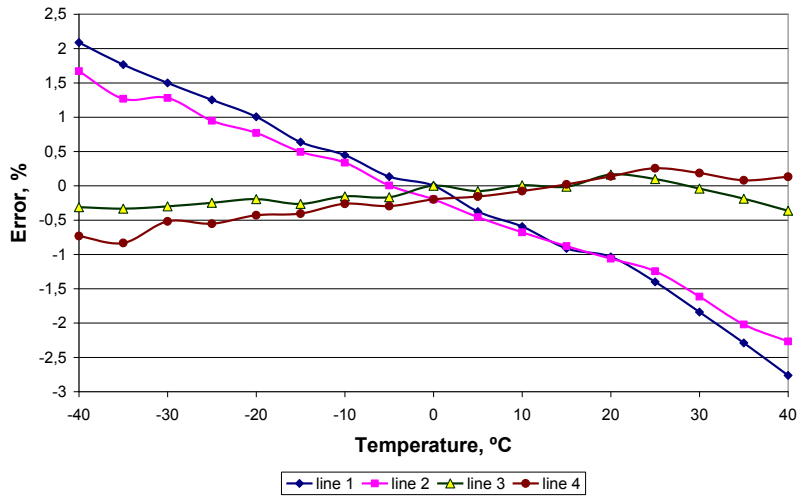

Fig. 6. Temperature error of LPG mass measurement for measuring system with RF sensor tuned for propane.

Diagrams for temperature dependencies of dielectric permittivity for propane (line 1) and butane (line 2) are presented in Fig. 5. Modeling results for temperature error of LPG mass measurement in measuring system with RF sensor tuned for propane mass measurement are in Fig. 6: line 1 - error of propane mass measurement without temperature correction; line 2 - error of butane mass measurement without temperature correction; line 3 - error of propane mass measurement with temperature correction $+0,06 \%$ per $1{ }^{\circ} \mathrm{C}$; line 4 - error of butane mass measurement with temperature correction $+0,06 \%$ per $1{ }^{\circ} \mathrm{C}$ (correction by temperature for propane).

In order to do diagrams in Fig. 6 values of dielectric permittivity are scaled (reduced to density value for propane at the temperature $0{ }^{\circ} \mathrm{C}$ ) through multiplication by coefficient $K$ that allows to present dielectric permittivity by density; it is used for tuning to a maximum if needed: 


$$
K=\rho_{l 0} /\left(\varepsilon_{l 0}-1\right)
$$

where $\rho_{l 0}=0,5297 \mathrm{~g} / \mathrm{cm}^{3}$ is density of propane at $0{ }^{\circ} \mathrm{C} ; \varepsilon_{l 0}=1,7148$ is dielectric permittivity of propane at $0{ }^{\circ} \mathrm{C}$; according to initial tuning $K=0,5297 /(1-1,7148)=0,7410$.

Errors of mass measurement for each temperature are calculated as a difference between scaled increase values of dielectric permittivity (related to density that is used at sensor operation) and real density (reference data); this difference is divided by $\rho_{l 0}=0,5297 \mathrm{~g} / \mathrm{cm}^{3}$ (density of propane at $0{ }^{\circ} \mathrm{C}$ ) and multiplied by $100 \%$. Such measuring system can measure mass of propane with error not more than $\pm 0,5 \%$ (line 3 ) and mass of butane with error not more than $1 \%$ (being tuned for propane, without taking into account errors because of inaccurate recalculation of calibration table).

Knowing the form of a reservoir containing LPG it is possible to determine the volume occupied by LPG liquid and gaseous phases. So, for a vertical reservoir with circular form filling in along its longitudinal axis, volumes $V_{l}$ and $V_{g}$ of LPG liquid and gaseous phases accordingly are: $V_{l}=h S ; V_{g}=V_{0}-V_{l}$, где $h$ - level of LPG liquid phase, $V_{0}$ - volume of a reservoir. Such a consideration is correct if clear interface exists between LPG liquid and gaseous phases. Then knowing densities $\rho_{l}$ and $\rho_{g}$ of LPG liquid and gaseous phases corresponding values of their masses can be determined: $M_{l}=\rho_{l} V_{l} ; M_{g}=\rho_{g} V_{g}$. If gradient of temperature is not high (not more than $\pm 3{ }^{\circ} \mathrm{C}$ ), for calculations temperature $t_{l}$ is chosen that is an averaged value of data of temperature sensors positioned in LPG liquid phase.

Increase $C_{s}$ of equivalent capacitance of RF sensor caused by the presence of LPG liquid and gaseous phases in a vertical reservoir is

$$
C_{s}(h)=C_{l} \times\left[\left(\varepsilon_{l}-1\right) \times h+\left(\varepsilon_{g}-1\right) \times\left(L_{1}-h\right)\right]
$$

where $\mathrm{C}_{l}$ is sensor electrical capacitance per $1 \mathrm{~m}$ in vacuum, $\left.\mathrm{C}_{l}=47 \mathrm{pF}\right) ; h$ is level of LPG liquid phase, filling in the sensor; $L_{1}$ is sensor length; $\varepsilon_{l}$ is dielectric permittivity of LPG liquid phase; $\varepsilon_{g}$ is dielectric permittivity of LPG gaseous (vapor) phase.

LPG mass in a reservoir is determined by the formula:

$$
M=V \times \rho_{l}+\left(V_{0}-V\right) \times \rho_{g}=S \times h \times \rho_{l}+S \times\left(L_{1}-h\right) \times \rho_{g}
$$

where $V_{0}$ - total volume of a reservoir; $V$ - volume of the liquid in a reservoir; $\rho_{l}, \rho_{g}-$ densities of LPG liquid and gaseous phases accordingly; $S$ - area of a reservoir crosssection, it is constant value for a vertical circular reservoir and is a function of $h$ in general case that is its value is changed along the height of a reservoir.

Formula (2) can be written so :

$$
M=S \times\left[h \times \rho_{l}+\left(L_{1}-h\right) \times \rho_{g}\right]
$$

Densities $\rho_{l}, \rho_{g}$ of LPG liquid and gaseous phases are proportional to the values of $\left(\varepsilon_{l}-1\right)$, $\left(\varepsilon_{g}-1\right)$ accordingly.

Dielectric permittivities of liquid and gaseous phases of propane and butane and of their mixtures within LPG compositions are calculated according to the formulas:

$$
\begin{aligned}
& \varepsilon_{l}=1+\rho_{l} /\left[K \times\left(1+T_{\mathrm{s}} \times t_{l}\right)\right] \\
& \varepsilon_{g}=1+\rho_{g} /\left[K \times\left(1+T_{\mathrm{s}} \times t_{l}\right)\right]
\end{aligned}
$$

where $K=\rho_{ж 0} /\left(\varepsilon_{ж 0}-1\right)=0,741 \mathrm{~g} / \mathrm{sm}^{3} ; T_{\mathrm{s}}-$ coefficient of temperature correction, $T_{\mathrm{s}}=$ 0,$0006 ; t_{l}$ is chosen that is an averaged value of data of temperature sensors positioned in LPG liquid phase.

It is done permissible check-up: if temperature from any sensor differs more than $3{ }^{\circ} \mathrm{C}$ from temperature values from adjacent sensors then data from this sensor are not taken into account.

After substitution of (4) and (5) to the formula (1) and joint processing of (1) and (3) it is received:

$$
M=S \times C_{\mathrm{s}} \times K \times\left(1+T_{\mathrm{s}} \times t_{l}\right) / C_{l}
$$

So, LPG total mass in a reservoir at various relationships of mass $M_{g}$ of gaseous phase and of mass $M_{l}$ of liquid phase and at different composition of components is determined by the formula (6) and depends only on $C_{s}$ and $t_{l}$. 
Coefficient $T_{\mathrm{s}}$ removes temperature error that is $0,06 \%$ per $1{ }^{\circ} \mathrm{C}$; it is much less tan temperature coefficient of density change for liquefied gases that is $0,26 \%$ per $1{ }^{\circ} \mathrm{C}$.

Area $S$ of a reservoir's cross-section is a constant value for a vertical reservoir. For reservoirs of other forms, in particular for horizontally-placed cylindrical reservoirs that are in gas tank trucks area $S$ is written by the table as the function of $C_{\mathrm{s}}$. Above received results are recalculated taking into account geometry of reservoirs.

\section{Radiofrequency sensors}

Electrical scheme with RF sensor that is capacitive sensor with coaxial position of its conductors, and electronic unit is shown in Fig. 7. Device at this scheme is auto oscillator. RF coaxial sensor serves as frequency-setting element of auto oscillator 1. Besides coaxial sensor electrical scheme contains also inductance 2. Presence of several dielectric supports that are teflon disks in particular, provides rigid mutual position of inner 3 and outer (metal tube) 4 conductors of the sensor. Only two such supports 5 and 6 are shown in Fig. 6. They have through holes for free liquid movement. For instance, these supports can be positioned at the distance $0,7 \mathrm{~m}$ from each other like in real devices.

Fig. 7. Scheme with RF sensor.

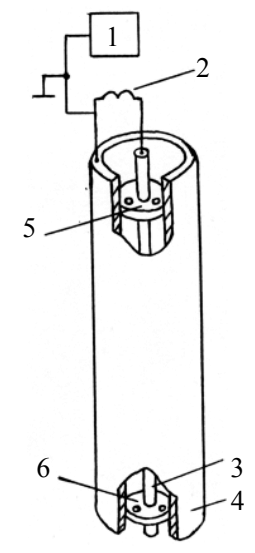

1 - autogenerator, 2 - inductance, 3 - inner conductor, 4 - metal tube,

5 and $6-$ dielectric supports.

In electronic unit of this measuring system it is done joint functional processing of output signals of RF sensor and of temperature sensors in order to determine LPG quantitative parameters.

\section{Position of measuring equipment in a gas tank truck}

Position of measuring equipment in a gas tank truck is shown in Fig. 8. System unit SB-5 (Fig. 3) is positioned in any suitable place (on the frame of a semi-trailer or on the back wall of the cabin). Mass meters Micro Motion ${ }^{\circledR}$ are positioned in the box with other equipment on pipelines for LPG liquid and gaseous phases. LPG RF mass sensor DZhS-7m (Fig. 2) for a reservoir is installed at lower or upper hatch of a reservoir. Many gas tank trucks don't have lower or upper hatch but they have lateral or end hatch for mechanical level sensor. Sensor DZhS-7B (Fig. 2) is installed in this case. Such sensor is assembly of two sensors DZhS-7m, one and second of these sensors are positioned at the angle $35^{\circ}$ up and down. This assembly operates as a single sensor called DZhS-7B. 
At the extended configuration the measuring system contains LPG pouring off unit contains usually a filter with gas-separator, mass meter and differential valve. Pouring off unit contains Coriolis mass meter, RF sensor of liquid boiling DZhS-7PTD and spherical tap with electrical driver regulated by controller. Because of absence of gas separator and differential valve reliability (no mechanical items) becomes higher and velocity of pouring off (operationwith lower step of pressure is provided) is increased. Supply of an assigned LPG quantity is provided by controller of the unit SB-7 that processes data from mass meter and controls electrical driver of the spherical tap. Sensor DZhS-7PTD measures LPG density, pressure and temperature in a pipeline and allows to provide pouring off regimes when LPG boiling is absent with guaranty. If non-condensated LPG vapour is present in a pipeline this sensor indicates presence of LPG boiling and the tap will not be opened. In this case vapour is needed to be removed to a plug or into a reservoir, at once after that LPG pouring off will start.

Repumping of LPG vapour phase may be needed under LPG pouring off through mass meter [7]. LPG pouring off is stopped in this case by covering the pipeline for LPG liquid phase. Then the pipeline for vapour coming back is opened and it is closed again after equilizing pressures. Duration of the stop is not more than 10 minutes. LPG mass sensor in a tank of a gas tank truck allows to measure mass of overpumped vapour with high accuracy.

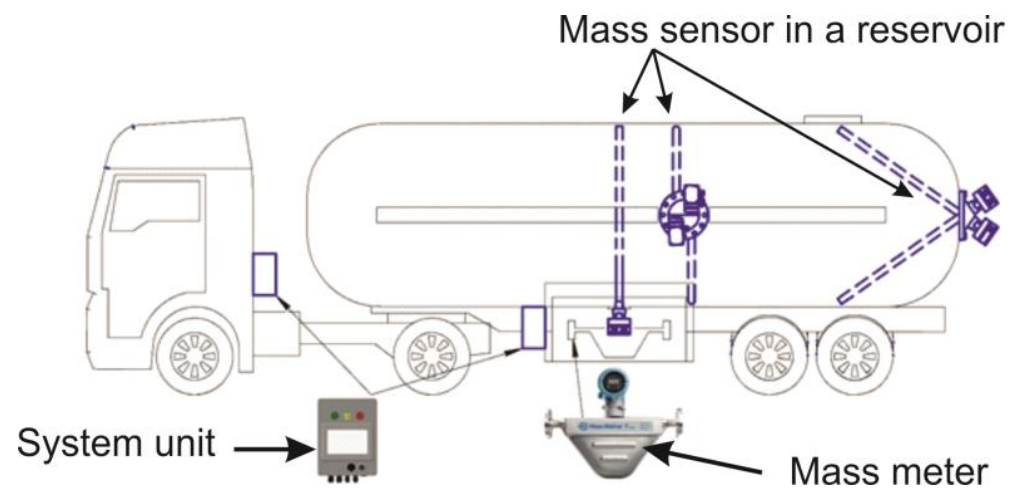

Fig. 8. Position of the equipment in a gas tank truck.

Nonmeasured rest is LPG volume that is located under working zone of the sensor. For example the lower end of the sensor is at the distance $70 \mathrm{~mm}$ from the bottom of a reservoir. LPG volume 470 litres and mass near $240 \mathrm{~kg}$ relate to this level for autocystern with the volume $36 \mathrm{~m}^{3}$. Under LPG pouring off lower this level data of the sensor don't change - level $70 \mathrm{~mm}$ and mass of LPG liquid phase near $240 \mathrm{~kg}$; big error of LPG mass measurement will appear. In these conditions mass of LPG vapour phase depends on LPG temperature ond composition and is within the limits from $70 \mathrm{~kg}$ to $600 \mathrm{~kg}$. LPG pouring off up to nonmeasured rest is not recommended in order to provide accurate measurement results.

\section{Calibration and check-up of the sensor in a reservoir}

Needed condition for accurate measurements is correct calibration table for a reservoir [7]. Calibration of the sensors DZhS-7m or DZhS-7B together with a reservoir where a sensor is installed is done via Coriolis flow meter at a gas tank truck. LPG poiring off (if a reservoir is calibrated at a gas tank truck together with mass meter) or filling in (if a reservoir is calibrated at another gas tank truck or at a gas filling station) of a reservoir through mass meter are done. Calibration is done on a low velicity of LPG overloading 
without interruptions. Measurement results come to a server simultaneously from a mass meter and a sensor in a reservoir. At joint processing of these data new graduation characteistics is received that is written into the spark-protection unit IZK-3. Check-up of a sensor in a reservoiris done similarly but interruptions of overloading at checked points are done. Advantages if this way: degasation of reservoirs and their removal from exploitation is not needed; calibration and check-up are done in a usual way of equipment operation and don't need a lot of time; presence of a check-up doing person at an object during check-up is not needed because data from a mass meter and a sensor in a reservoir come to a server in-line; software for a server allows to do automatically reports by results of checkup; inclination of a tank of a gas tank truck is taken into account; real matching of a sensor to a geometry of a reservoir is taken into account. Check-up of a sensor in a reservoir may be done via scales. A gas tank truck together with a sensor are installed on scales for this purpose. Another gas tank truck (filled in by LPG) is neded to be put near the cheched-up truck and connected to the truck on the scales by hoses for LPG liquid and gaseous phases. One of gas tank trucks sould be equipped by a pump. Overloading of LPG and reception of dsata of the scales and the sensor in a reservoir are done. Before the check-up calibration of a sensor in a tank of a gas tank truck via mass meter should be done.

\section{Conclusion}

Suggested technical decisions allow to provide total counting of LPG in transport tanks, to exclude fully ability of non-sanctioned LPG overloads. Sensors don't have mechanical elements resulting in their high high reliability at severe exploitation conditions. Measurement results come on-line to a server, archives and reports are done according to executed trade operations.

\section{References}

1. S.I. Gausner, S.S. Kivilis, A.P. Osokina, A.N. Pavlovskiy, Measurement of mass, volume and density (Publ. house of standards, Moscow, 528 p. (in Russian) (1982)

2. B.S. Rachevskiy, Liquefied petroleum gases (Oil and gas Publ. house, Moscow, 640 p.) (in Russian) (2009)

3. E.I. Zorja, A.L. Yakovlev, S.V. Larionov, Determination of liquefied petroleum gas mass under reception, storage and delivery to consumers (Nedra Publ. House, Moscow, 197 p.) (in Russian) (2012)

4. A.S. Sovlukov, V.I. Tereshin, Measurement of liquefied petroleum gas quantity in a tank by radio-frequency techniques, IEEE Transactions on Instrumentation and Measurement, v. 53, 4, pp. 1255-1261(2004)

5. A.S. Sovlukov, V.I. Tereshin, A radio-frequency method of measuring the quantitative parameters of liquefied natural gas in tanks, Measurement Techniques, v. 48, 10, pp. 1038-1044 (2005)

6. A.S. Sovlukov, V.I. Tereshin, Measurement of the quantity of liquefied petroleum gas in a tank, Measurement Techniques, v. 49, 2, pp. 40-42 (2006)

7. A.S. Sovlukov, V.I. Tereshin, Radiofrequency measurement of liquefied petroleum gas mass in a reservoir, Sensors and systems, 12, pp. 41-45 (in Russian) (2012)

8. N.P. Bogorodizkiy, Yu.M. Volokobinskiy, A.A. Vorobyov, B.M. Tareev, Theory of dielectrics (Energiya Publ. house, Moscow-S.-Petersburg, 344 p. (in Russian) (1965) 